\title{
Preserving executive functions in nondominant frontal lobe glioma surgery: an intraoperative tool
}

\author{
*Guglielmo Puglisi, PhD, ${ }^{2,3}$ Tommaso Sciortino, MD, ${ }^{1}$ Marco Rossi, MD, ${ }^{1}$ Antonella Leonetti, ${ }^{2,3}$ \\ Luca Fornia, PhD, ${ }^{2}$ Marco Conti Nibali, MD, ${ }^{1}$ Alessandra Casarotti, ${ }^{3}$ Federico Pessina, MD, ${ }^{3}$ \\ Marco Riva, MD, ${ }^{3,4}$ Gabriella Cerri, PhD, ${ }^{2}$ and Lorenzo Bello, MD ${ }^{1}$
}

\begin{abstract}
${ }^{1}$ Neurosurgical Oncology Unit, Department of Oncology and Hemato-Oncology, Università degli Studi di Milano, and Humanitas Research Hospital, IRCCS, Milano, Italy; ${ }^{2}$ Laboratory of Motor Control, Department of Medical Biotechnology and Translational Medicine, Università degli Studi di Milano, and Humanitas Research Hospital, IRCCS, Milano, Italy; ${ }^{3}$ Neurosurgical Oncology Unit, Humanitas Research Hospital, IRCCS, Milano, Italy; and 4Department of Medical Biotechnology and Translational Medicine, Università degli Studi di Milano, and Humanitas Research Hospital, IRCCS, Milano, Italy
\end{abstract}

\begin{abstract}
OBJECTIVE The goal of surgery for gliomas is maximal tumor removal while preserving the patient's full functional integrity. At present during frontal tumor removal, this goal is mostly achieved, although the risk of impairing the executive functions (EFs), and thus the quality of life, remains significant. The authors investigated the accuracy of an intraoperative version of the Stroop task (iST), adapted for intraoperative mapping, to detect EF-related brain sites by evaluating the impact of the iST brain mapping on preserving functional integrity following a maximal tumor resection.
\end{abstract}

METHODS Forty-five patients with nondominant frontal gliomas underwent awake surgery; brain mapping was used to establish the functional boundaries for the resection. In 18 patients language, praxis, and motor functions, but not EFs (control group), were mapped intraoperatively at the cortical-subcortical level. In 27 patients, in addition to language, praxis, and motor functions, EFs were mapped with the iST at the cortical-subcortical level (Stroop group). In both groups the EF performance was evaluated preoperatively, at 7 days and 3 months after surgery.

RESULTS The iST was successfully administered in all patients. Consistent interferences, such as color-word inversion/ latency, were obtained by stimulating precise white matter sites below the inferior and middle frontal gyri, anterior to the insula and over the putamen, and these were used to establish the posterior functional limit of the resection. Procedures implemented with iST dramatically reduced the EF deficits at 3 months. The EOR was similar in Stroop and control groups.

CONCLUSIONS Brain mapping with the iST allows identification and preservation of the frontal lobe structures involved in inhibition of automatic responses, reducing the incidence of postoperative EF deficits and enhancing the further posterior and inferior margin of tumor resection.

https://thejns.org/doi/abs/10.3171/2018.4.JNS18393

KEYWORDS awake surgery; cognitive control; executive functions; glioma; intraoperative brain mapping; resection; oncology

$\mathrm{T}$ HE treatment of gliomas consists of surgery and associated chemo- and radiotherapy. At present, the extent of resection (EOR) is one of the main factors affecting the natural course of the disease (survival, progression-free-survival, and time to malignant transformation). Brain mapping (BM) allows brain tumor resection to be performed in accordance with functional rather than purely anatomical boundaries, reaching total and even supratotal resection while maintaining functional integrity. ${ }^{35,40}$ The preservation of patients' neurofunctional integrity can be assured by increasing the number of neurological functions to be mapped and preserved during surgery. At present, BM allows for monitoring of the main motor, language, and also praxis functions.,13,30 However, the evaluation of executive functions $(\mathrm{EFs})^{1,10,12}$-i.e., the complex set of abilities allowing control of cognitive processes and behavior to reach specific goals-remains not fully explored..$^{32}$ In everyday life the EFs play an essential

ABBREVIATIONS BM = brain mapping; DES = direct electrical stimulation; $E F=$ executive function; $E O R=$ extent of resection; iST = intraoperative version of the Stroop task; HF = high-frequency; LF = low-frequency; LGG = low-grade glioma.

SUBMITTED February 9, 2018. ACCEPTED April 23, 2018.

INCLUDE WHEN CITING Published online September 28, 2018; DOI: 10.3171/2018.4.JNS18393.

* G.P. and T.S. share first authorship of this work. 
role in contextual adjustment and social interactions, as in those conditions in which it is required to focus on a specific behavior despite distractions or temptations; to make a plan for the future; or to voluntarily switch from one activity to another. From an anatomical-functional perspective, a complex frontal corticostriatal network connecting prefrontal cortices and the basal ganglia $6,7,16,19,25,28,31$ (the frontostriatal pathway) is suggested to control the EFs. Patients with lesions affecting this network (mainly the orbital frontal cortex and the lateral prefrontal cortex) show impulsive behavior ${ }^{39}$ and difficulties in inhibiting irrelevant stimuli.

A retrospective analysis of our patients (control group; see Methods) who underwent gross-total or supratotal resection for nondominant frontal glioma from 2014 through 2015-i.e., before the introduction of the Stroop task in $\mathrm{BM}$-highlighted a relevant prevalence of postsurgical EF deficits, such as inappropriate or perseverative behaviors and limitations in performing tasks requiring high attentive control/effort. These deficits are usually underestimated by clinicians, even though they severely impair the patient's ability to work, study, and develop and maintain appropriate social relations. ${ }^{18}$ This evidence raises the need to implement the current BM with tasks designed to reduce postsurgical $\mathrm{EF}$ disabilities, while performing a total or, if feasible, a supratotal resection of the tumor to achieve the main functional and oncological goals. To this end, the main challenges consist of developing a test feasible for surgery, and thus one easily administered intraoperatively, and at the same time highly accurate and selective for the different EF components (selective attention, inhibitory control, working memory, speed processing, and mental flexibility ${ }^{12}$ ).

We developed a simplified intraoperative version of the Stroop task (iST), a well-known task usually used to assess crucial components of EFs. ${ }^{23,37}$ A modified version of the same task has already been used for cortical mapping, but its feasibility for subcortical mapping ${ }^{38}$ has not been reported and its effect on functional outcome has not been systematically assessed.

We evaluated the feasibility and accuracy of the iST on preserving the EFs; these objectives were met by comparing the EF outcomes of patients who underwent surgery while using the iST (Stroop group) with the EF outcomes of a matched group of patients who underwent resection without the iST (control group).

\section{Methods}

\section{Patients Selection}

Forty-five patients with gliomas undergoing tumor resection in an asleep-awake-asleep procedure with the aid of BM were included in the study. The sample was composed of 2 groups: the Stroop group (enrolled from 2016 to 2017) was composed of 27 patients who underwent standard BM plus the iST, and the control group (enrolled from 2014 to 2015) was composed of 18 patients who underwent standard BM without the iST.

Patients in both groups fulfilled the following inclusion criteria: 1) a nondominant frontal lobe glioma treated with a total or supratotal resection that was planned; 2) absence of language and visual deficits; 3) normal scores on a presurgical Stroop test (details to follow); 4) matched demographics and tumor characteristics (Table 1). All patients gave written informed consent to the surgical and mapping procedure, which followed the principles outlined in the World Medical Association's "Declaration of Helsinki." The study was performed with strict adherence to the routine procedure adopted for surgical tumor removal.

\section{Neuroradiological Assessment}

Preoperative MRI was performed using a Philips Medical Systems Intera 3-T scanner (Koninklijke Philips N.V.), and images acquired for lesion morphological characterization and volumetric assessment. The MRI protocol included: axial 3D fluid-attenuated inversion-recovery (3D FLAIR) images, post-gadolinium administration 3D T1weighted fast-field-echo images, and apparent diffusion coefficient calculated using diffusion-weighted images.

Volumetric analysis was used to define tumor volume. The mean lesion volume in each group of patients is reported in Table 1. The mean preoperative volume was not different between the two groups (degrees of freedom = 42; $\mathrm{p}=0.429)$.

The lesion volume was computed onto FLAIR volumetric sequences with manual segmentation using the iPlan cranial software suite (Brainlab AG) by 2 investigators (T.S. and M.R.). FLAIR hyperintense signal abnormalities were included in the lesion load for low-grade gliomas (LGGs) and reported in centimeters cubed. Patients underwent both an immediate (within 48 hours) and a 3-month postoperative MRI (volumetric FLAIR and post-gadolinium administration T1-weighted imaging) to estimate the EOR. The EOR corresponded to the percentage of the volume resected with respect to the preoperative volume: (preoperative volume - postoperative volume)/(preoperative volume). The EORs were classified as follows: total resection, $\mathrm{EOR}=100 \%$; subtotal resection, EOR $=90 \%-100 \%$; and supratotal resection, EOR > $100 \% .^{2}$ Postoperative diffusion-weighted MRI scans were also obtained to check for ischemic damage.

\section{Assessment of EFs}

Preoperative and Postoperative Assessments

All patients (Stroop and control groups) underwent extensive preoperative (1 week before surgery) and postoperative (7 days and 3 months after surgery) neuropsychological assessment. A selected group of tests assessed the different components of EFs (Table 2).

\section{Intraoperative Assessment}

A modified version of the Stroop test was used during surgery in 27 patients (Stroop group) to assess the EFs. The complete version of the Stroop test is extensive and composed of 3 subtasks $^{8}$ in which the patient is instructed to answer as fast and as accurately as possible to the relevant stimulus attribute: in the first subtask the patient is asked to read a list of color names (red, blue, or green), while in the second subtask the patient is asked to name the color of a series of colored dots (red, blue, or green). These subtasks allow for the exclusion of reading 
TABLE 1. Demographic and clinical features of the patients at baseline

\begin{tabular}{|c|c|c|c|}
\hline Characteristics & Stroop Group & Control Group & $p$ Value \\
\hline No. of patients & 27 & 18 & \\
\hline Sex, no. $(\%)$ & & & 0.374 \\
\hline Male & $11(40.7)$ & $10(55.6)$ & \\
\hline Female & $16(59.3)$ & $8(44.4)$ & \\
\hline Mean age, yrs $( \pm$ SD) & $37.8 \pm 11.9$ & $37.8 \pm 14.4$ & 0.99 \\
\hline $\begin{array}{l}\text { Median yrs of education } \\
\text { (range) }\end{array}$ & $15(13-17)$ & $13(8-17)$ & 0.235 \\
\hline $\begin{array}{l}\text { Seizure at presentation, } \\
\text { no. }(\%)\end{array}$ & $18(66.7)$ & $11(61.1)$ & 0.758 \\
\hline \multicolumn{4}{|l|}{ No. of AEDs (\%) } \\
\hline 1 & $14(51.85)$ & $12(66.7)$ & \\
\hline 2 & $7(25)$ & $4(22.2)$ & \\
\hline $\begin{array}{l}\text { Median KPS score at } \\
\text { presentation (range) }\end{array}$ & $100(80-100)$ & $100(90-100)$ & 0.818 \\
\hline \multicolumn{4}{|l|}{$\begin{array}{l}\text { Histology (WHO 201622), } \\
\text { no. (\%) }\end{array}$} \\
\hline WHO grade II & $18(66.7)$ & $7(38.9)$ & \\
\hline Astrocytoma & 10 & 3 & \\
\hline Oligodendroglioma & 8 & 4 & \\
\hline WHO grade III & $9(33.3)$ & $10(55.6)$ & \\
\hline Astrocytoma & 5 & 5 & \\
\hline Oligodendroglioma & 4 & 5 & \\
\hline WHO grade IV & & $1(5.6)$ & \\
\hline Glioblastoma & 0 & 1 & \\
\hline IDH-1/IDH-2 mutation (\%) & $25(92.6)$ & $17(94.4)$ & 0.807 \\
\hline $\begin{array}{l}\text { Mean lesion volume, } \mathrm{cm}^{3} \\
\quad( \pm \mathrm{SD})\end{array}$ & $33.5 \pm 46.9$ & $44.2 \pm 38.3$ & 0.429 \\
\hline $\begin{array}{l}\text { Supratotal resection + } \\
\text { GTR, no. (\%) }\end{array}$ & $23(85.2)$ & $18(100)$ & 0.138 \\
\hline Supratotal resection & 21 & 15 & 0.721 \\
\hline GTR & 2 & 3 & \\
\hline Subtotal resection, no. (\%) & $4(14.8)$ & 0 & \\
\hline Median EOR, \% (range) & $100(83.3-100)$ & $100(100-100)$ & 0.099 \\
\hline
\end{tabular}

AED = antiepileptic drug; GTR = gross-total resection; IDH= isocitrate dehydrogenase; KPS = Karnofsky Performance Scale; WHO = World Health Organization.

or perceptual difficulties. The third subtask (color-word subtask) specifically evaluates the EFs: a series of color words printed in an incongruent hue (e.g., blue printed in a red hue) is presented to patients who must report the hue color. In order to accomplish the task patients must inhibit their automatic tendency to read the written color (blue) to report the color the word is presented in (red).$^{11}$ The color-word subtask ${ }^{37}$ is highly sensitive for the identification of frontal lobe lesions, resulting in delayed responses and increased errors; thus, an adapted version of the colorword subtask (iST) was coupled with BM to map the EFs during surgery. One word at a time (font size: $80 \mathrm{pt}$ ) was presented at the center of a white background on a 9.7inch monitor; at the word's appearance, the patient had to name, as fast as possible, the hue (blue, green, red) of
TABLE 2. Neuropsychological assessment of EFs

\begin{tabular}{|c|c|}
\hline Test & Assessed Abilities \\
\hline $\begin{array}{l}\text { Raven progres- } \\
\text { sive matrices }\end{array}$ & $\begin{array}{l}\text { Nonverbal reasoning: to reason by analogy to make } \\
\text { inference }\end{array}$ \\
\hline $\begin{array}{l}\text { Attentive matri- } \\
\quad \operatorname{ces}^{36}\end{array}$ & $\begin{array}{l}\text { Selective attention: to selectively react to certain } \\
\text { stimuli while suppressing attention to other non- } \\
\text { relevant concurrent ones }\end{array}$ \\
\hline $\begin{array}{l}\text { Trail making } \\
\text { test }^{17}\end{array}$ & $\begin{array}{l}\text { Divided attention: to switch between or pay atten- } \\
\text { tion to two simultaneous subtasks }\end{array}$ \\
\hline Verbal fluency ${ }^{26}$ & $\begin{array}{l}\text { Lexical access speed \& verbal monitoring: to } \\
\text { generate specific words \& to suppress irrelevant } \\
\text { responses \& repetition }\end{array}$ \\
\hline $\begin{array}{l}\text { Digit span } \\
\text { backward } 24\end{array}$ & $\begin{array}{l}\text { Working memory: to manipulate selected informa- } \\
\text { tion \& mentally working with it }\end{array}$ \\
\hline Stroop task ${ }^{8}$ & $\begin{array}{l}\text { Selective attention \& inhibitory control: to resist } \\
\text { interference, cognitive flexibility, \& inhibition of } \\
\text { overlearned responses in favor of unusual ones }\end{array}$ \\
\hline
\end{tabular}

the word ("blue," "green," "red"). The task was performed during or in the absence of stimulation. The correctness of the patient's verbal response was reported to the neurosurgeon, and then the next word was shown (about 2 seconds for each word) (Fig. 1).

\section{Surgical Procedure}

Surgery, guided by the BM and performed according to functional boundaries, was aimed at obtaining a supratotal resection whenever feasible. The craniotomy exposed the tumor area and a limited amount of surrounding tissue. In the awake phase of the procedure, cortical mapping was used to identify the cortical safe entry zone, while subcortical mapping was performed to define the functional boundaries of the tumor. Once eloquent subcortical tracts surrounding the tumor were identified and the tumor was functionally disconnected, the mass was removed after induction of general anesthesia. In patients treated through 2015 (the control group), standard $\mathrm{BM}$ was used to preserve language, ${ }^{5}$ motor, ${ }^{5}$ and praxis $^{30}$ functions, while from 2016 standard BM was combined with the iST to identify cortical-subcortical eloquent sites also for the EFs (the Stroop group). In this group, patients were stimulated with direct electrical stimulation (DES) while performing the iST. DES was applied with a lowfrequency $(\mathrm{LF}-\mathrm{DES})$ paradigm $(60 \mathrm{~Hz})$ or a high-frequency (HF-DES) paradigm (TO-5, repetition rate $3 \mathrm{~Hz}$ ) set at the same current intensity adopted for language mapping $(2.75 \pm 0.93 \mathrm{~mA})$. In 4 patients, during LF-DES language and motor mapping over the ventral premotor area, despite the fact that the current intensity was increased up to the maximal values routinely applied (5-6 mA), no responses were obtained but some afterdischarges were detected by electrocorticography. To avoid the risk of seizures, the LFDES was replaced with HF-DES for the mapping..$^{29}$ In 4 patients, both the LF and HF paradigm were consecutively applied to the same sites.

\section{Intraoperative Mapping and Stimulation Sites}

$\mathrm{BM}$ implemented with the iST was video recorded 


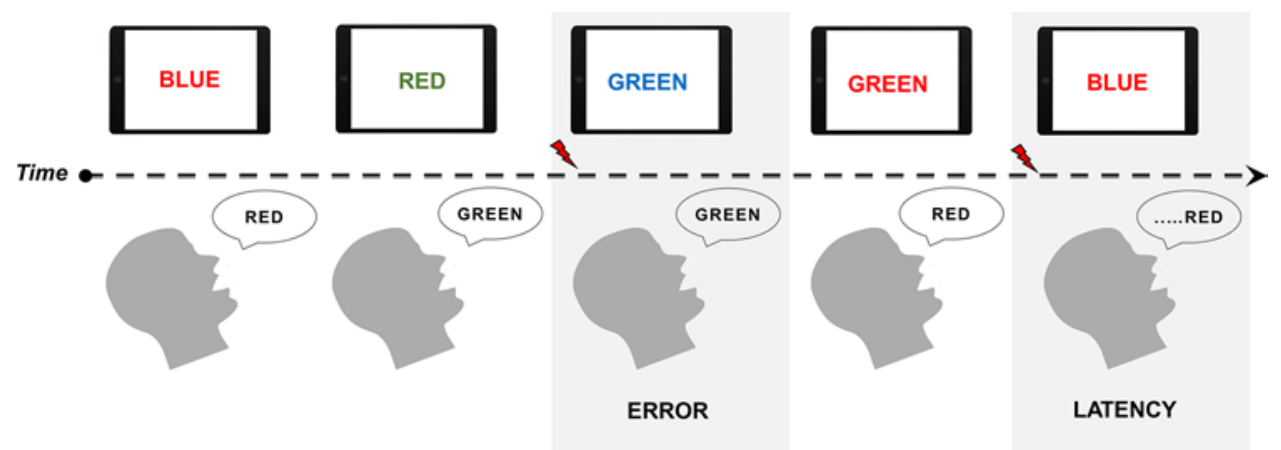

FIG. 1. The iST intraoperative task consists of an adapted version of the Stroop task; one word at time was presented on a 9.7-inch monitor and the patient was asked to name, as fast as he/she could, the color of the ink of the observed word presented on the monitor. The response of the patient is reported in the bubble below each illustrated monitor. The red lightning represents the time at which the DES was applied over the investigated site. The duration of the stimulus was 2 seconds. Figure is available in color online only.

and reviewed offline by surgeons and neuropsychologists to verify the stimulation sites and the corresponding responses. A stimulation site (cortical or subcortical) was considered positive when its stimulation impaired the task performance at least 3 nonconsecutive times ${ }^{14,27}$ and specifically when, during DES, patients uttered the written word instead of the color (error) or when the correct response was given with a delay $>1$ second (latency). During the iST, the patients' verbal responses were reported in real time to the neurosurgeon by the neuropsychologist, fully blinded to the neuroanatomical location of the stimulation site. Positive sites for the iST were also assessed with naming, semantic, and motor tasks to exclude the possible attribution of the interferences during the iST to language or motor disturbances.

\section{Normalization Procedure and Statistical Analysis}

The locations of positive sites were recorded by neuronavigation (Curve, Brainlab AG), correcting brain shift with intraoperative ultrasound (Hitachi Aloka Medical, Ltd.) at the end of the subcortical mapping procedure, just before the resection of the tumor. The recorded positive sites were verified offline on the video recordings and normalized in an MNI (Montreal Neurological Institute) space using an affine transformation implemented in SPM8 software. ${ }^{3}$

The efficacy of the iST was evaluated 7 days and at 3 months after the surgery by comparison between Stroop and control groups of the number of subjects showing EF deficits in at least one test of the neuropsychological assessment. To avoid an incorrect assignment of the onset of postoperative deficits to the surgical treatment itself, only the occurrence of new EF deficits was considered. Patients' continuous variables were reported as mean $\pm \mathrm{SD}$ or median and were compared with a Student t-test. Categorical variables were compared with the Fisher exact test. We considered two-sided p values less than 0.05 to be statistically significant. Statistical analysis was performed with SPSS Statistics 22.0 for Mac software (IBM Corp.).

\section{Results}

The aim of the study was to evaluate the feasibility and the impact of a new intraoperative tool, a modified version of the Stroop task (iST), during BM in preserving postoperative EFs during maximal brain tumor resection.

\section{Feasibility}

The iST was successfully administered intraoperatively-by the neuropsychologist or in its execution by the patient-in all 27 patients without difficulties. Specifically, the iST was delivered with the same intraoperative tool used for the administration of language tasks (a 9.7-inch tablet device), and the duration of its administration was an average of 4 minutes (range 3-5 minutes) at the cortical level and 7 minutes (range 5-8 minutes) at the subcortical level, with a global impact on the total duration of mapping of about 11 minutes (range 8-13 minutes). The average overall duration of the BM in the awake phase was 23 minutes (range 20-27 minutes).

\section{Intraoperative Findings}

When delivered on positive sites for iST, DES impaired the task performance, specifically inducing color-word inversion (error $65 \%$ ) or, less frequently, a delayed response (latency 35\%) but never a lack of response. Different DES protocols delivered (HF-DES and/or LF-DES) on the same site produced the same errors.

Although no positive sites for iST were found when stimulating the cortex of the inferior, medial, or superior frontal gyrus, a significant number of positive sites (mean 4 [range 3-5]) were found subcortically, mainly clustering in a discrete subcortical area within the white matter under the inferior frontal gyrus and medial frontal gyrus, in front of the anterior insula, and lateral to the head of the caudate nucleus, passing over the putamen and the anterior thalamus to reach the cingulum (Fig. 2). The iST positive sites were not responsive to the other functions, evaluated during subcortical BM (language, motor, and praxis tasks).

\section{Neuropsychological Outcome}

Figure 3 summarized, for both the Stroop and control groups, the percentage of patients with a subnormal score in at least one test of the neuropsychological battery at 

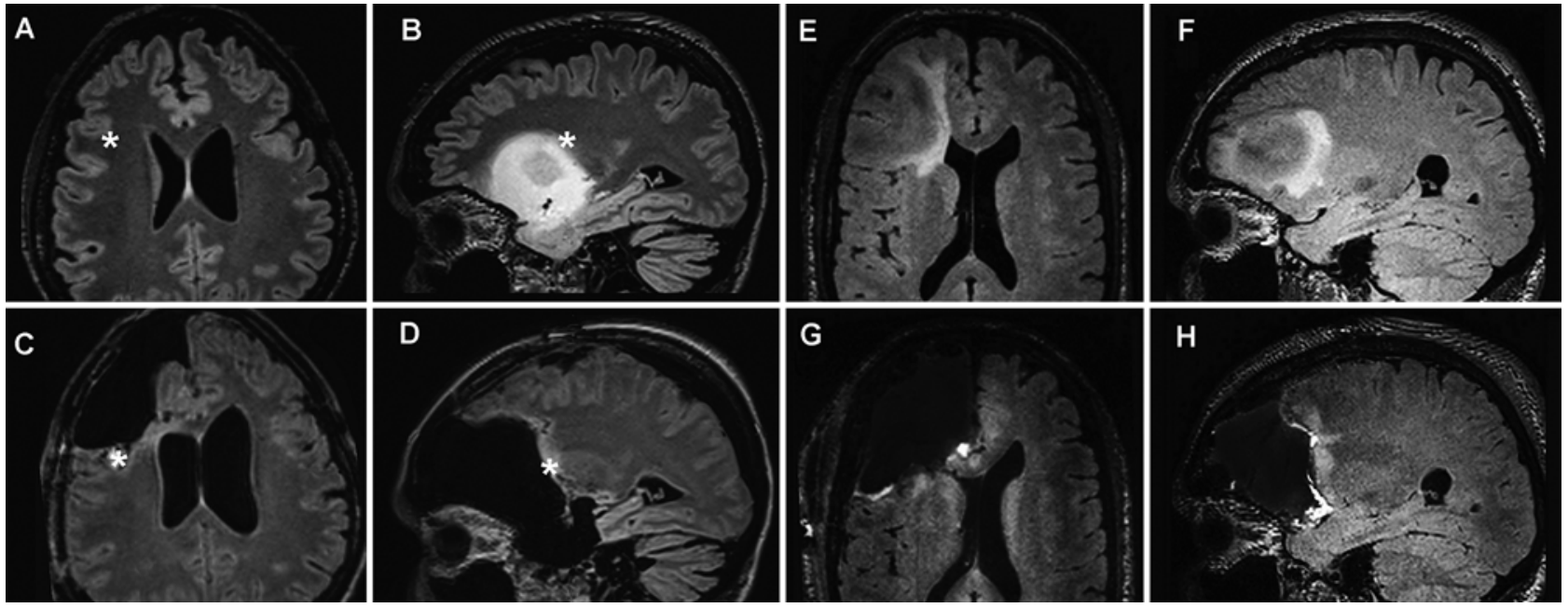

FIG. 2. Representative MR images of 2 cases in which the LGG was located in the nondominant frontal lobe. A-D: Stroop group (preoperative axial and sagittal FLAIR [A and B]; postoperative axial and sagittal FLAIR [C and D]) images. E-H: Control group (preoperative axial and sagittal FLAIR [E and F]; postoperative axial and sagittal FLAIR [G and H]) images. The white asterisks in A-D represent the location of a positive site that gave interferences during DES while the patient was performing the iST; the resection was subsequently stopped at this site.

all time points (presurgery, 7 days postoperatively, and 3 months postoperatively). No difference in EF deficits between Stroop $(14.8 \%)$ and control $(27.8 \%)(\mathrm{p}=0.449)$ groups were observed before surgery and at 7 days after surgery. The Stroop group patients had a lower incidence of $\mathrm{EF}$ deficits $(51.9 \%)$ than the control group patients $(77.8 \%)(\mathrm{p}=0.07)$. The difference between the groups further increased at 3 months after surgery, due to a decrease of the incidence of EF deficits in the Stroop group compared to the control group ( $22.2 \%$ and $61.1 \%$, respectively) $(\mathrm{p}=0.01)$.

When specifically analyzing the subcomponents of EFs compromised in the long term (3 months), it emerged that the few patients in the Stroop group showing EF deficits were actually impaired only in one subcomponent (verbal fluency in $86 \%$ [ 6 of 7 patients]). On the other hand, nearly half $(54 \%)$ of the control group patients showing EF deficits were compromised in more than one subcomponent (digit span backward, Stroop test, and verbal fluency). Interestingly, the incidence of deficits in the digit span backward testing was significantly higher in the control group than the Stroop group (33\% vs 0\% [p = 0.004]).

\section{Effect on EOR and Intraoperative Surgical Strategy}

In both groups, a supratotal resection, largely exceeding the tumor volume, was performed. The median EORs in both groups were not statistically different (Stroop group $100 \%$ [range $83.3 \%-100 \%$; control group 100\% [range $100 \%-100 \%$ ]; $\mathrm{p}=0.099$ ), providing evidence that, overall, the iST did not significantly impact EOR. The surgical relevance in iST administration involves a higher precision in functional border identification during the procedure. In the absence of the iST, the posterior margin of the resection was determined by subcortical sites that were positive for motor or, less frequently, language responses, or the margin was based on anatomical landmarks (the ventricle or the insular cortex). Administration of the iST increased the accuracy in establishment of the posterior and deep border (Fig. 2A-D). The residual tumor in the 3 Stroop group patients with a subtotal resection coincided with positive motor cognition sites, establishing the posterior limit of the resection.

\section{Discussion}

Resection of brain tumors involving BM is the gold standard for preserving patients' functional integrity and maximizing the EOR. At present, BM is efficient in helping to preserve several motor, praxis, and language functions. ${ }^{5,14,30}$ Unfortunately, the lack of an adequate intraoperative task has thus far prevented the mapping of $\mathrm{EFs},{ }^{1,10,12}$ and this has resulted in postoperative deficits, as suggested by the analysis of patients admitted to our unit in 2015, treated with resection conducted with standard BM. Specifically, at 3 months after surgery in about $61 \%$ of these patients, neuropsychological assessment revealed the occurrence of deficits on at least one of the tests as-

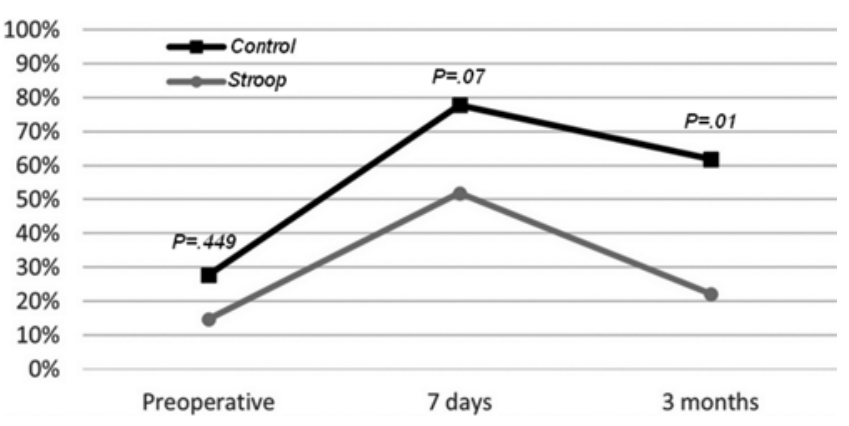

FIG. 3. Trend of EF deficits. Prevalence (as a percentage) of EF deficits measured 7 days before surgery (i.e., Preoperative), 7 days after surgery, and at 3 months after in the patients from the Stroop (gray line) or the control (black line) group. $P$ represents the significance of the difference in EF deficits between the two groups for each time point. 
sociated with EFs. Such deficits are often underestimated despite their effect on patients' lives, which can be significantly impaired in routine daily activities. Recent neuroimaging studies of lesions suggest that EFs are controlled by a system of fibers running in the inferior frontal lobe. 9,20 This evidence led, in our hypothesis, to the idea that postoperative EF deficits might be caused by the intraoperative resection of fibers running in frontal subcortical pathways and acting on the prefrontal cortices. To test this hypothesis, we evaluated the feasibility and efficacy of an intraoperative task designed to localize and preserve the EFrelated structures during surgery. To this end, the Stroop task, usually adopted to assess certain crucial components of EFs, ${ }^{23,37}$ was adapted for intraoperative mapping to allow the monitoring of EFs every 1-2 seconds, the main requirement of the procedure.

We demonstrated that the iST is a feasible and accurate tool. It is an efficient tool for administration during awake mapping, in that it is easy to administer without the introduction of additional tools and it is easy to take for the patients. Moreover, analysis of the anatomical distribution of the positive sites for the iST revealed high accuracy for the task in that its responses specifically related to the iST clustered in a discrete site within the white matter of the frontal lobe and resulted in disruption of the ability to correctly perform the task time-locked to DES, all features required in an intraoperative task to be relevant for surgery. To further support the specificity of the iST for EF mapping, there is the evidence that the same sites that were positive for the iST failed to elicit either motor responses (hand or mouth) or interference with praxis or language function.

The intraoperative feasibility and the accuracy of iST was paralleled by the clinical efficacy in preserving EFs as demonstrated by the neuropsychological assessments in which we observed a significant reduction of EF deficits at 7 days postsurgery and, above all, in the long term (i.e., 3 months after surgery) in the Stroop group patients as compared to the control group patients. Moreover, the analysis of the individual subcomponents of the EFs in both groups suggests that the iST is particularly effective at preserving working memory, whereas its main limitation appears to be that it is not able to preserve lexical access speed.

The involvement of subcortical frontal tracts in EFs, suggested by the literature, ${ }^{2,15,21,33,34}$ might be supported by our findings showing that the iST eloquent area is located along fibers running from the inferior frontal gyrus and medial frontal gyrus to the anterior insula, lateral to the head of the caudate nucleus and over the putamen, targeting the cingulum. Evidence correlating subcortical tract abnormalities with emotional or inhibitory control disturbances in autism suggests the involvement of frontostriatal or frontoanterior thalamic connections, possibly involving insular connectivity, in EF deficits. ${ }^{20}$ Further intraoperative studies are mandatory to address this issue.

From a surgical point of view, the iST seems to be a valid tool to increase the accuracy of identifying the tumor's functional boundaries located in the deep, posterior white matter of the nondominant frontal lobe, which allows for a safe resection at this level according to functional-and not only anatomical-landmarks. Of surgical relevance is that introduction of the iST does not lead to a decrease in the EOR, in that a large (supratotal) resection was possible in both groups of patients, but furthermore it allowed us to perform extensive resections while preserving functional integrity of EFs, achieving the goals of the surgical treatment of brain tumors, particularly the LGGs, that require large resections.

\section{Conclusions}

Our data suggest that the iST is a feasible and reliable tool to be used intraoperatively to identify and preserve the networks underlying the EFs and thus to reduce, with high specificity, the incidence of the higher-order cognitive disorders associated with supratotal resection of nondominant frontal lobe tumors impairing the quality of life of patients.

\section{Acknowledgments}

This work was supported by grant from Associazione Italiana per la Ricerca sul Cancro (AIRC) to L.B.

\section{References}

1. Alvarez JA, Emory E: Executive function and the frontal lobes: a meta-analytic review. Neuropsychol Rev 16:17-42, 2006

2. Aron AR, Behrens TEJ, Smith S, Frank MJ, Poldrack RA: Triangulating a cognitive control network using diffusionweighted magnetic resonance imaging (MRI) and functional MRI. J Neurosci 27:3743-3752, 2007

3. Ashburner J, Friston KJ: Unified segmentation. Neuroimage 26:839-851, 2005

4. Basso A, Capitani E, Laiacona M: Raven's coloured progressive matrices: normative values on 305 adult normal controls. Funct Neurol 2:189-194, 1987

5. Bello L, Riva M, Fava E, Ferpozzi V, Castellano A, Raneri F, et al: Tailoring neurophysiological strategies with clinical context enhances resection and safety and expands indications in gliomas involving motor pathways. Neuro Oncol 16:1110-1128, 2014

6. Braver TS, Barch DM, Gray JR, Molfese DL, Snyder A: Anterior cingulate cortex and response conflict: effects of frequency, inhibition and errors. Cereb Cortex 11:825-836, 2001

7. Bunge SA, Ochsner KN, Desmond JE, Glover GH, Gabrieli JD: Prefrontal regions involved in keeping information in and out of mind. Brain 124:2074-2086, 2001

8. Caffarra P, Vezzadini G, Dieci F, Zonato F, Venneri A: Una versione abbreviata del test di Stroop: dati normativi nella popolazione italiana. Nuova Riv Neurol 12:111-115, 2002

9. Catani M, Dell'Acqua F, Budisavljevic S, Howells H, Thiebaut de Schotten M, Froudist-Walsh S, et al: Frontal networks in adults with autism spectrum disorder. Brain 139:616-630, 2016

10. Chan RCK, Shum D, Toulopoulou T, Chen EYH: Assessment of executive functions: review of instruments and identification of critical issues. Arch Clin Neuropsychol 23:201-216, 2008

11. Cohen JD, Dunbar K, McClelland JL: On the control of automatic processes: a parallel distributed processing account of the Stroop effect. Psychol Rev 97:332-361, 1990

12. Diamond A: Executive functions. Annu Rev Psychol 64:135-168, 2013

13. Duffau H: The usefulness of the asleep-awake-asleep glioma surgery. Acta Neurochir (Wien) 156:1493-1494, 2014 
14. Duffau H, Capelle L, Sichez N, Denvil D, Lopes M, Sichez $\mathrm{JP}$, et al: Intraoperative mapping of the subcortical language pathways using direct stimulations. An anatomo-functional study. Brain 125:199-214, 2002

15. Forstmann BU, Jahfari S, Scholte HS, Wolfensteller U, van den Wildenberg WPM, Ridderinkhof KR: Function and structure of the right inferior frontal cortex predict individual differences in response inhibition: a model-based approach. J Neurosci 28:9790-9796, 2008

16. Garavan H, Ross TJ, Stein EA: Right hemispheric dominance of inhibitory control: an event-related functional MRI study. Proc Natl Acad Sci U S A 96:8301-8306, 1999

17. Giovagnoli AR, Del Pesce M, Mascheroni S, Simoncelli M, Laiacona M, Capitani E: Trail making test: normative values from 287 normal adult controls. Ital J Neurol Sci 17:305309,1996

18. Knouse LE, Barkley RA, Murphy KR: Does executive functioning (EF) predict depression in clinic-referred adults? EF tests vs. rating scales. J Affect Disord 145:270-275, 2013

19. Konishi S, Nakajima K, Uchida I, Kikyo H, Kameyama M, Miyashita Y: Common inhibitory mechanism in human inferior prefrontal cortex revealed by event-related functional MRI. Brain 122:981-991, 1999

20. Langen M, Leemans A, Johnston P, Ecker C, Daly E, Murphy $\mathrm{CM}$, et al: Fronto-striatal circuitry and inhibitory control in autism: findings from diffusion tensor imaging tractography. Cortex 48:183-193, 2012

21. Lipszyc J, Levin H, Hanten G, Hunter J, Dennis M, Schachar R: Frontal white matter damage impairs response inhibition in children following traumatic brain injury. Arch Clin Neuropsychol 29:289-299, 2014

22. Louis DN, Perry A, Reifenberger G, von Deimling A, Figarella-Branger D, Cavenee WK, et al: The 2016 World Health Organization Classification of Tumors of the Central Nervous System: a summary. Acta Neuropathol 131:803-820, 2016

23. MacLeod CM: Half a century of research on the Stroop effect: an integrative review. Psychol Bull 109:163-203, 1991

24. Monaco M, Costa A, Caltagirone C, Carlesimo GA: Erratum to: forward and backward span for verbal and visuo-spatial data: standardization and normative data from an Italian adult population. Neurol Sci 36:345-347, 2015

25. Munakata Y, Herd SA, Chatham CH, Depue BE, Banich MT, O'Reilly RC: A unified framework for inhibitory control. Trends Cogn Sci 15:453-459, 2011

26. Novelli G, Papagno C, Capitani E, Laiacona M, Vallar G, Cappa SF: Tre test clinici di ricerca e produzione lessicale. Taratura su sogetti normali. Arch Psicol Neurol Psichiatr 47:477-506, 1986

27. Ojemann G, Ojemann J, Lettich E, Berger M: Cortical language localization in left, dominant hemisphere. An electrical stimulation mapping investigation in 117 patients. J Neurosurg 71:316-326, 1989

28. Ouellet J, McGirr A, Van den Eynde F, Jollant F, Lepage M, Berlim MT: Enhancing decision-making and cognitive impulse control with transcranial direct current stimulation (tDCS) applied over the orbitofrontal cortex (OFC): A randomized and sham-controlled exploratory study. J Psychiatr Res 69:27-34, 2015

29. Riva M, Fava E, Gallucci M, Comi A, Casarotti A, Alfiero $\mathrm{T}$, et al: Monopolar high-frequency language mapping: can it help in the surgical management of gliomas? A comparative clinical study. J Neurosurg 124:1479-1489, 2016

30. Rossi M, Fornia L, Puglisi G, Leonetti A, Zuccon G, Fava E, et al: Assessment of the praxis circuit in glioma surgery to reduce the incidence of postoperative and long-term apraxia: a new intraoperative test. J Neurosurg [epub ahead of print February 23, 2018. DOI: 10.3171/2017.7.JNS17357]

31. Rubia K, Russell T, Overmeyer S, Brammer MJ, Bullmore ET, Sharma T, et al: Mapping motor inhibition: conjunctive brain activations across different versions of go/no-go and stop tasks. Neuroimage 13:250-261, 2001

32. Satoer D, Visch-Brink E, Dirven C, Vincent A: Glioma surgery in eloquent areas: can we preserve cognition? Acta Neurochir (Wien) 158:35-50, 2016

33. Schmahmann JD, Pandya DN: The complex history of the fronto-occipital fasciculus. J Hist Neurosci 16:362-377, 2007

34. Seghete KLM, Herting MM, Nagel BJ: White matter microstructure correlates of inhibition and task-switching in adolescents. Brain Res 1527:15-28, 2013

35. Smith JS, Chang EF, Lamborn KR, Chang SM, Prados MD, Cha $S$, et al: Role of extent of resection in the long-term outcome of low-grade hemispheric gliomas. J Clin Oncol 26:1338-1345, 2008

36. Spinnler H, Tognoni G: Taratura e standardizzazione italiana di test neuropsicologici. Ital J Neurol Sci Suppl 8:1987

37. Stuss DT, Floden D, Alexander MP, Levine B, Katz D: Stroop performance in focal lesion patients: dissociation of processes and frontal lobe lesion location. Neuropsychologia 39:771-786, 2001

38. Wager M, Du Boisgueheneuc F, Pluchon C, Bouyer C, Stal V, Bataille B, et al: Intraoperative monitoring of an aspect of executive functions: administration of the Stroop test in 9 adult patients during awake surgery for resection of frontal glioma. Neurosurgery 72 (2 Suppl Operative):ons169-ons181, 2013

39. Winstanley CA: The orbitofrontal cortex, impulsivity, and addiction: probing orbitofrontal dysfunction at the neural, neurochemical, and molecular level. Ann N Y Acad Sci 1121:639-655, 2007

40. Yordanova YN, Moritz-Gasser S, Duffau H: Awake surgery for WHO Grade II gliomas within "noneloquent" areas in the left dominant hemisphere: toward a "supratotal" resection. Clinical article. J Neurosurg 115:232-239, 2011

\section{Disclosures}

The authors report no conflict of interest concerning the materials or methods used in this study or the findings specified in this paper.

\section{Author Contributions}

Conception and design: Puglisi, Sciortino, Rossi, Leonetti, Casarotti, Bello. Acquisition of data: Puglisi, Sciortino, Rossi, Leonetti, Conti Nibali, Casarotti, Pessina, Riva, Bello. Analysis and interpretation of data: Puglisi, Sciortino, Rossi, Leonetti, Fornia, Cerri, Bello. Drafting the article: Puglisi, Sciortino, Leonetti, Fornia, Cerri, Bello. Critically revising the article: Rossi, Leonetti, Fornia, Conti Nibali, Pessina, Riva, Cerri, Bello. Statistical analysis: Puglisi, Sciortino. Study supervision: Cerri, Bello. Direction and execution of the surgical procedure and the intraoperative brain mapping: Bello.

\section{Supplemental Information}

\section{Previous Presentations}

Some preliminary results of the study were presented at the following: European Workshop on Cognitive Neuropsychology, Brixen, Italy, January 22-27, 2017 (poster); EANS Meeting, Venice, Italy, October 1-5, 2017 (ePoster); 12th European Low Grade Glioma Network, Bilbao, Spain, June 1-3, 2017 (oral presentation); and 6th International Society of Intraoperative Neurophysiology Congress, Seoul, Korea, October 30 to November 4, 2017 (oral presentation).

\section{Correspondence}

Guglielmo Puglisi: Università degli Studi di Milano and Humanitas Research Hospital, Milan, Italy.guglielmo.puglisi@gmail.com. 